\title{
The impact of genetic factors and testing on operative indications and extent of surgery for aortopathy
}

Elizabeth L. Norton, $\mathrm{MS},{ }^{\mathrm{a}}$ and Bo Yang, $\mathrm{MD}, \mathrm{PhD}^{\mathrm{b}}$

Feature Editor Note-Great strides have been made in understanding the genetic basis of thoracic aortic disease. While many cases do not have a clear heritable pattern, for those that do, knowledge of the genetic basis may have direct implications for management of both the patient and their relatives. Numerous causative genes have been identified for both syndromic and nonsyndromic heritable thoracic aortic disease, and it is expected that many more have yet to be discovered. The patterns of aortic disease associated with each mutation are being investigated, and from this, we are learning that patient management should be gene-specific. For example, the diameter threshold for elective aortic resection and the extent of resection should be tailored not just to the named syndrome but also to the specific gene mutation, when it is known. In the accompanying review, Yang and colleagues explain the current state of genetic testing and its future in the management of both patients and families with heritable thoracic aortic disease.

\section{Leora B. Balsam, MD}

Thoracic aortic aneurysm affects 5.9 in 100,000 people a year, ${ }^{1}$ whereas acute aortic dissection affects 5 to 30 million people a year ${ }^{2}$ with $20 \%$ to $30 \%$ of patients with a thoracic aortic aneurysm or dissection (TAAD) having an underlying genetic predisposition. ${ }^{3,4}$ To date, variants in more than 30 genes have been implicated in TAAD, ${ }^{5}$ with definitive or strong evidence for 11 genes. ${ }^{4}$ Heritable thoracic aortic disease (HTAD) can be syndromic_-associated with additional systemic features, such as Marfan syndrome (MFS) or Loeys-Dietz syndrome (LDS) —or nonsyndromic, as with ACTA2, MYLK, and MYH11 mutations. ${ }^{6}$ Although all variants in causative genes are known as heritable thoracic disease, each gene mutation behaves differently, resulting in varying clinical courses. Identification of patients at risk and understanding of the pathology and clinical course of each mutation can facilitate clinical decision-making and

From ${ }^{\mathrm{a} C r e i g h t o n}$ University School of Medicine, Omaha, Neb; and ${ }^{\mathrm{b}}$ Department of Cardiac Surgery, Michigan Medicine, Ann Arbor, Mich.

Dr Yang is supported by the National Heart, Lung, and Blood Institute of National Institutes of Health K08HL130614, R01HL141891, and R01HL151776, and the Phil Jenkins and Darlene \& Stephen J. Szatmari Funds.

Received for publication Jan 13, 2021; accepted for publication Jan 14, 2021; available ahead of print April 1, 2021.

Address for reprints: Bo Yang, MD, PhD, Frankel Cardiovascular Center, 1500 East Medical Center Dr, 5155, Ann Arbor, MI 48109 (E-mail: boya@med.umich.edu). JTCVS Open 2021;6:15-23

2666-2736

Copyright $(C 2021$ The Authors. Published by Elsevier Inc. on behalf of The American Association for Thoracic Surgery. This is an open access article under the CC BY-NCND license (http://creativecommons.org/licenses/by-nc-nd/4.0/).

https://doi.org/10.1016/j.xjon.2021.01.013

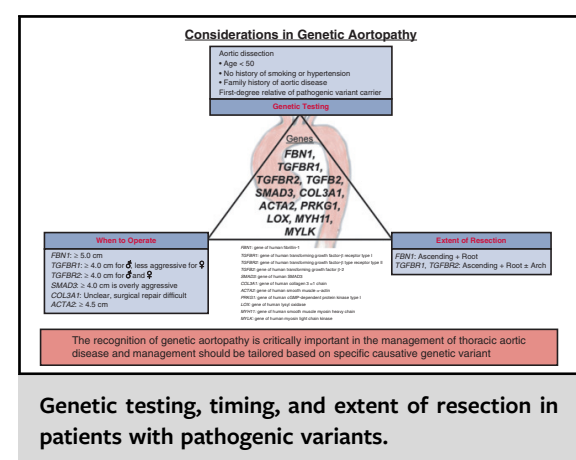

CENTRAL MESSAGE

The recognition of genetic aortopathy is critically important in the management of aortic disease and management should be tailored based on specific causative genetic variant.

See Commentary on page $\mathbf{2 4}$.

improve outcomes in TAAD. In this review, we uniquely (1) discuss the strategy of managing each aortopathy based on the individual gene mutations instead of the named syndromes; (2) incorporate the latest publications, such as sex differences in patients with TGFBRI mutations and the difference in aortic events between SMAD3 mutations and $T G F B R 1$ and TGFBR 2 mutations despite being under the LDS umbrella term; (3) discuss the management of these patients from a surgical standpoint, both when to operate based on specific gene mutations and how much to resect in the initial elective surgery or emergent acute type A dissection repair to prevent future complications and reoperations; and (4) provide a comprehensive list of Clinical Laboratory Improvement Amendments-certified genetic tests, respective company, and what genes are analyzed with each test respectively.

\section{GENES OF HTAD}

The majority of causative variants categorized as HTAD have been identified in genes that encode proteins of the extracellular matrix, involved in vascular smooth muscle contraction and metabolism, and transforming growth factor- $\beta$ signaling pathways that are essential to the integrity of the aortic wall. ${ }^{5,7}$ MFS, LDS, and vascular EhlersDanlos syndrome (vEDS) primarily comprise syndromic 
HTAD with mutations in FBN1, TGFBR1 and TGFBR2, and COL3A1, respectively, with other syndromic TAAD having been reported, although less prevalent and with less cardiovascular burden. ${ }^{8-10}$ Other systemic features of syndromic TAAD often include involvement of the musculoskeletal, ocular, cutaneous, and integumentary systems among others such as joint laxity, long bone overgrowth, ectopia lentis, pectus deformity, scoliosis, skin striae, etc.

MFS is an autosomal-dominant connective tissue disorder that results from mutations in FBNl, the gene for fibrillin-1 (an essential component of the extracellular matrix), which results in skeletal (long bone overgrowth, joint laxity, vertebral column deformity), ocular (lens displacement, myopia), and cardiovascular (aortic root aneurysm and dissection, mitral valve prolapse) abnormalities, among others. The cardiovascular abnormalities, specifically aortic dissection and rupture, are the leading cause of morbidity and mortality. ${ }^{11}$

LDS is an autosomal-dominant connective tissue disorder that results from mutations in TGFBR1, TGFBR2, $S M A D 3, T F G B 2$, and TGFB3, which encode proteins of the TGF- $\beta$ signaling pathway, a critical pathway in blood vessel development and vascular maintenance. ${ }^{3,10}$ Patients with LDS exhibit many overlapping features with MFS (joint laxity, pectus, joint laxity, long bone overgrowth) and vEDS (easy bruising, thin skin); however, craniofacial features such as ocular hypertelorism, bifid uvula/cleft palate, and cervical spine instability are unique to LDS. ${ }^{3}$ In addition, aortic disease in LDS tends to be more severe and aggressive, with aortic dissection and rupture at younger ages and smaller aortic diameters and more widespread vasculopathy, with aneurysms being more widespread and not limited to the aorta as in MFS., $3,4,12$

vEDS is an autosomal-dominant connective tissue disorder that results from mutations in COL $3 A 1$, the gene for proalpha1 chains of type III collagen (the major structural component of large blood vessels). ${ }^{8}$ vEDS has the worst prognosis of all EDS subtypes and is characterized by abnormalities of the skin and joints as well as arterial, intestinal, and uterine fragility, with arterial dissection or rupture being the most common cause of death, including the thoracic or abdominal aorta. ${ }^{13}$

Nonsyndromic HTAD, isolated cardiovascular findings without systemic features, is a heterogeneous cohort of patients in which the underlying genetic causes include mutations in ACTA2, MYH11, MYLK, PRKG1, LOX, FOXE3, MAT2A, MFAP5, BGN, ELN, NOTCH1, and COL5A1, among others, in addition to FBN1, FBN2, TGFBR1, TGFBR2, SMAD3, TFGB2, TGFB3, and COL3A1, which can be syndromic or nonsyndromic, ${ }^{3}$ with definitive or strong for variants in ACTA2, COL3A1, FBN1, MYH11, SMAD3, TGFB2, TGFBR1, TGFBR2, MYLK, LOX, and $P R K G 1^{4}$ (Table 1). In patients with nonsyndromic TAAD,
TABLE 1. Genes with strong or definitive evidence associated with syndromic and nonsyndromic heritable thoracic aortic disease

\begin{tabular}{ll}
\hline \multicolumn{1}{c}{$\begin{array}{c}\text { Heritable thoracic aortic } \\
\text { disease }\end{array}$} & \multicolumn{1}{c}{ Associated genes } \\
\hline $\begin{array}{l}\text { Syndromic } \\
\text { Marfan syndrome }\end{array}$ & $\begin{array}{l}\text { FBN1 } \\
\text { Loeys-Dietz syndrome } \\
\text { Vascular Ehlers-Danlos } \\
\quad \text { syndrome }\end{array}$ \\
COL3A1 \\
Nonsyndromic & $A C T A 2, M Y L K, M Y H 11, P R K G 1, L O X$ \\
\hline
\end{tabular}

up to $20 \%$ have a strong family history-termed familial thoracic aortic aneurysm and dissection, with up to $30 \%$ of nonsyndromic HTAD having a causative pathogenic variant in one of the known HTAD-related genes. The underlying gene in which the pathogenic mutation is located can predispose to a certain phenotype, for example, patients with pathogenic variants in PRKGl present with aortic dissection relatively early in life and at smaller aortic diameters. ${ }^{14}$

Bicuspid aortic valve (BAV) is a heterogenous disorder with associated aortopathy and despite established heritability, specific causative variants remain unsubstantiated. Genes with the most extensive evidence are NOTCH1, GATA factors (GATA4, GATA5, GATA6), KROX22, and ROBO4; however, all of the variants combined account for a small percentage of BAV cases and the mutations are yet to be identified in the majority of BAV cases. ${ }^{15-20}$ Mutations in NOTCHI and ROBO4 have the most evidence and could be associated with BAV; however, clinical decisions are not based on genetic variants in BAV since the association is not strong and there are no genetic tests. Patients with BAV can be classified clinically into 2 subtypes based on valvulopathy and aortopathy as we presented previously ${ }^{16}$ : (a) malignant form-root aneurysm and aortic insufficiency-and (b) benign form-ascending aneurysm and aortic stenosis and could be treated with a more aggressive and conservative approach, respectively. Patients with BAV can be managed based on phenotype as well as family history. For patients with BAV we recommend an elective operation for thoracic aortic aneurysm (root/ascending) at $5 \mathrm{~cm}$ for patients with a family history of aortic dissection.

\section{GENETIC TESTING}

Advances over the past 20 years have led to increased discovery and understanding of genetic and molecular mechanisms involved in TAAD, with the number of causative variants increasing with time; however, many still lack evidence or remain undiscovered. Although a majority of TAAD are degenerative in nature with hypertension as the major risk factor, genetic predisposition is the second major 
risk factor. ${ }^{7,21}$ Genetics are a crucial piece in determining clinical management of TAAD. Guidelines for when to pursue surgical intervention are primarily based on aortic diameter criteria; however, diameter ranges indicating a need for surgical repair vary based on etiology. The American Heart Association/American College of Cardiology guidelines $^{22}$ recommend that patients with genetically mediated aneurysms undergo elective surgical repair at an ascending or aortic root diameter of 4.0 to $5.0 \mathrm{~cm}$, depending on the condition. However, underlying genetic etiology often is unknown at time of presentation with TAAD, and the identification of pathogenic genetic variants have the potential to improve management and direct treatment strategies. A recent study by our group ${ }^{23}$ identified pathogenic variants in $10.8 \%$ of patients with a history of thoracic aortic dissection, with pathogenic variants identified in COL3A1, FBN1, LOX, PRKG1, SMAD3, and TGFBR2. In addition, age $<50$ years, no history of hypertension, and family history of aortic disease were significantly associated with pathogenic variant carriers ${ }^{23}$; therefore, young patients $(<50$ years old) with a family history and without hypertension should undergo genetic testing.

Similarly, Verhagen and colleagues ${ }^{24}$ recommend genetic testing in patients with a thoracic aortic aneurysm $\geq 45 \mathrm{~mm}$ or dissection and (1) age at diagnosis $<50$ years, or (2) age at diagnosis 50-60 years and no hypertension, or (3) positive family history, of (4) presence of syndromic features. ${ }^{24}$ If the patient has a pathogenic variant, either syndromic or nonsyndromic, then their family should be tested. In nonsyndromic HTAD, first- and second-degree relatives of patients with familial nonsyndromic HTAD and firstdegree relatives of patients with sporadic nonsyndromic HTAD should be screened. ${ }^{25}$ HTAD is a genetically heterogeneous disease: clinical manifestations overlap with many different gene mutations, and current sequencing techniques make it possible to sequence multiple genes within a short period of time at an affordable cost; therefore, testing of aortopathy gene panel should be conducted. Current aortopathy panels vary in genes they analyze, analyzing 4-52 genes depending on the company (Table 2). Milewicz and Regalado ${ }^{3}$ suggest genetic panels include some or all of 16 genes known to predispose to TAAD: ACTA2, $B G N$, COL3A1, FBN1, FOXE3, LOX, MAT2A, MFAP5, MYH11, MYLK, PRKG1, SMAD3, TGFB2, TGFB3, TGFBR1, TGFBR2. ACTA2, FBN1, MYH11, SMAD3, TGFBR1, and TGFBR2 are included in $>90 \%$ of available panels (Table 3). If a high-risk, young patient returns a negative panel, additional testing such as whole-exome sequencing, genome-wide screening, ${ }^{26}$ and copy-number vibration anal$\mathrm{ysis}^{27}$ may be indicated. In addition, testing could result in identification of a variant of unknown significance or a pathogenic variant in a gene not yet identified. Even if all genetic testing is negative, all first-degree family members should undergo aortic imaging to rule out thoracic aortic disease, ${ }^{23}$ because there are many pathogenic variants not discovered yet. Clinical genetic testing may help to prevent devastating events, such as thoracic aortic dissections and death, for patients and family members of pathogenic variant carriers who are high risk but have yet to develop the aortopathy.

\section{IMPLICATIONS OF POSITIVE GENETIC TESTING}

Genetic testing can return results indicating identification of (1) a pathogenic variant (variant in the gene identified is known to causative), (2) a variant of unknown significance (variant in known HTAD-gene that remains unclear if it is disease causing), and (3) no variant identified in the panel tested (does not guarantee absent risk as mutation could be present in genes not tested). ${ }^{28}$ Identification of a pathogenic variant in an HTAD gene can occur in a variety of settings and directly impacts the clinical decision making in the patient carrying the variant as well as the patient's first-degree relatives. In a young patient who presents with a thoracic aortic aneurysm with a family history of aortic disease or systemic features of syndromic TAAD, identification of a pathogenic variant can change surveillance and threshold diameter criteria for prophylactic surgical repair to prevent aortic dissection or rupture and lead to identification of TAAD in first-degree relatives, potentially leading to surveillance and prevention of catastrophic aortic events. However, it is important to use only well-validated genes for decision-making in the clinical setting, especially as new genes are identified. ${ }^{29}$

If a pathogenic variant is identified in $F B N 1$ and the patient is diagnosed with MFS, an echocardiogram is recommended at time of diagnosis, 6 months thereafter, and then annually to assess aortic root and ascending aorta diameters. $^{22}$ Prophylactic surgical repair of the aortic root or ascending aorta is indicated at an aortic diameter of $5.0 \mathrm{~cm}$ and smaller diameters if there is rapid growth $(>0.5 \mathrm{~cm} /$ year), family history of aortic dissection at a diameter $<5.0 \mathrm{~cm}$, of if there is significant aortic regurgitation. ${ }^{22,30}$

If a pathogenic variant is identified in TGFBRl, $T G F B R 2$, TGFB2, or SMAD3 and the diagnosis of LDS is made then complete aortic imaging should be performed at time of initial diagnosis and 6 months thereafter, similar to patients with MFS. However, patients with LDS should have an annual magnetic resonance imaging from the cerebrovascular circulation to the pelvic circulation as the arterial aneurysms in patients with LDS can be widespread. ${ }^{22}$ The American Heart Association/American College of Cardiology guidelines recommend prophylactic aortic repair when the aortic root or ascending aortic diameter is $\geq 4.2 \mathrm{~cm}$ on transesophageal echocardiogram and $\geq 4.4 \mathrm{~cm}$ on computed tomography or magnetic resonance imaging. ${ }^{22}$ Prophylactic surgical repair of the aorta in adult patients with LDS is reasonable when the aortic root or 
TABLE 2. Genetic testing for heritable thoracic aortic disease

\begin{tabular}{|c|c|c|c|c|}
\hline & Name & $\begin{array}{l}\text { Number } \\
\text { of genes }\end{array}$ & Genetic panel genes & Turnaround time \\
\hline 1 & $\begin{array}{l}\text { Invitae Aortopathy Comprehensive } \\
\text { Panel* }\end{array}$ & 24 & $\begin{array}{l}\text { ACTA2, CBS, COL3A1, COL5A1, COL5A2, EFEMP2, FBN1, } \\
\text { FBN2, FLNA, FOXE3, MED12, MYH11, MYLK, NOTCH1, } \\
\text { PLOD1, PRKG1, SKI, SLC2A10, SMAD3, SMAD4, TGFB2, } \\
\text { TGFB3, TGFBR1, TGFBR2 }\end{array}$ & $\begin{array}{r}\text { 10-21 calendar days } \\
\text { (14 d on average) }\end{array}$ \\
\hline 2 & ARUP Aortopathy Panel & 21 & $\begin{array}{l}\text { ACTA2, CBS, COL3A1, COL5A1, COL5A2, EFEMP2, FBN1, } \\
\quad F B N 2, \text { FLNA, LOX, } \dagger M Y H 11, \text { MYLK, PLOD1, PRKG1, SKI, } \\
\quad \text { SLC2A10, SMAD3, SMAD4, TGFB2, TGFB3, TGFBR1, TGFBR2 }\end{array}$ & $3-6 \mathrm{wk}$ \\
\hline 3 & $\begin{array}{l}\text { OHSU Knight Diagnostic } \\
\text { Laboratories Familial Aneurysm } \\
\text { and Aortopathy Panel }\end{array}$ & 31 & $\begin{array}{l}\text { ACTA2, ADAMTS2, B3GALT6, B4GALT7, CBS, CHST14, COL1A1, } \\
\text { COL1A2, COL3A1, COL5A1, COL5A2, FBN1, FBN2, FKBP14, } \\
\text { FLNA, MED12, MYH11, MYLK, NOTCH1, PLOD1, PRDM5, } \\
\text { SKI, SLC2A10, SLC39A13, SMAD3, TGFB2, TGFB3, TGFBR1, } \\
\text { TGFBR2, TNXB, ZNF469 }\end{array}$ & \\
\hline 4 & Blueprint Genetics Aorta Panel & 52 & 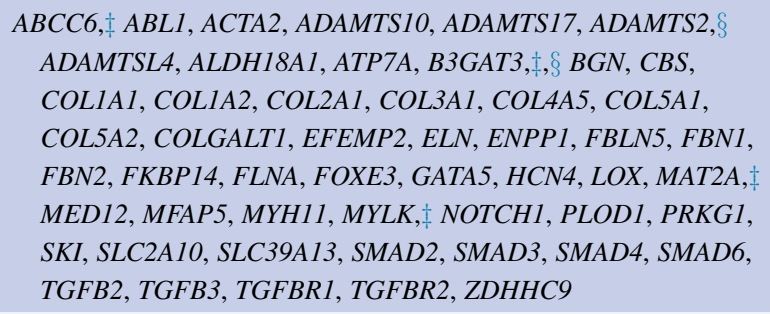 & $4 \mathrm{wk}$ \\
\hline 5 & $\begin{array}{l}\text { labcorp GeneSec: Cardio-Familial } \\
\text { Aortopathy Panel }\end{array}$ & 10 & $\begin{array}{l}\text { ACTA2, COL3A1, FBN1, MYH11, MYLK, SLC2A10, SMAD3, } \\
\quad \text { TGFB2, TGFBR1, TGFBR2 }\end{array}$ & $18-24 \mathrm{~d}$ \\
\hline 6 & GeneDx Marfan/TAAD Panel & 26 & $\begin{array}{l}\text { ACTA2, BGN, CBS, COL3A1, COL5A1, COL5A2, FBN1, FBN2, } \\
\text { FLNA, LOX, MAT2A, MED12, MFAP5, MYH11, MYLK, } \\
\text { NOTCH1, PRKG1, SKI, SLC2A10, SMAD2, SMAD3, SMAD4, } \\
\text { TGFB2, TGFB3, TGFBR1, TGFBR2 }\end{array}$ & $4 \mathrm{wk}$ \\
\hline 7 & AmbryGenetics TAADNext & 35 & $\begin{array}{l}\text { ACTA2, BGN, CBS, CHST14, COL1A1, COL1A2, COL3A1, } \\
\text { COL5A1, COL5A2, EFEMP2, FBN1, FBN2, FKBP14, FLNA, } \\
\text { FOXE3, LOX, MAT2A, MED12, MFAP5, MYH11, MYLK, } \\
\text { NOTCH1, PLOD1, PRDM5, PRKG1, SKI, SLC2A10, SMAD3, } \\
\text { SMAD4, TGFB2, TGFB3, TGFBR1,TGFBR2, TNXB, ZNF469 }\end{array}$ & $14-21 \mathrm{~d}$ \\
\hline 8 & $\begin{array}{l}\text { PreventionGenetics Familial } \\
\text { Thoracic Aortic Aneurysm and } \\
\text { Dissection Panel }\end{array}$ & 17 & $\begin{array}{l}\text { ACTA2, COL3A1, FBN1, FOXE3, LOX, MAT2A, MFAP5, MYH11, } \\
\text { MYLK, NOTCH1, PRKG1, SMAD3, SMAD4, TGFB2, TGFB3, } \\
\text { TGFBR1, TGFBR2 }\end{array}$ & $18 \mathrm{~d}$ on average \\
\hline 9 & $\begin{array}{l}\text { Collagen Diagnostic Lab Arterial } \\
\text { Aneurysm Panel }\end{array}$ & 25 & $\begin{array}{l}\text { ACTA2, BGN, CBS, COL1A1, COL3A1, FBN1, FBN2, FOXE3, } \\
\text { LOX, MAT2A, MFAP5, MYH11, MYLK, NOTCH1, PLOD3, } \\
\text { PRKG1, SKI, SLC2A10, SMAD2, SMAD3, SMAD4, SMAD6, } \\
\text { TGFB2, TGFB3, TGFBR1, TGFBR2 }\end{array}$ & \\
\hline 10 & $\begin{array}{l}\text { FulgentGenetics Marfan Syndrome } \\
\text { and TAAD Panel }\end{array}$ & 33 & $\begin{array}{l}\text { ACTA2, BGN, CBS, COL3A1, COL5A1, COL5A2, EFEMP2, ELN, } \\
\text { FBN1, FBN2, FLNA, FOXE3, HCN4, LOX, MAT2A, MED12, } \\
\text { MFAP5, MYH11, MYLK, NOTCH1, PLOD1, PRKG1, SKI, } \\
\text { SLC2A10, SMAD2, SMAD3, SMAD4, SMAD6, TAB2, TGFB2, } \\
\text { TGFB3, TGFBR1, TGFBR2 }\end{array}$ & $3-5 \mathrm{wk}$ \\
\hline 11 & $\begin{array}{l}\text { EGL Genetics Marfan Syndrome, } \\
\text { TAAD, and Related Disorders } \\
\text { Panel }\end{array}$ & 17 & $\begin{array}{l}\text { ACTA2, CBS, COL3A1, COL5A1, COL5A2, FBN1, FBN2, FLNA, } \\
\text { MED12, MYH11, MYLK, SKI, SLC2A10, SMAD3, TGFB2, } \\
\quad \text { TGFBR1, TGFBR2 }\end{array}$ & $6 \mathrm{wk}$ \\
\hline 12 & $\begin{array}{l}\text { Transgenomic Marfan Syndrome and } \\
\text { Aortic Aneurysm Panel }\end{array}$ & 4 & ACTA2, FBN1, TGFBR1, TGFBR2 & $4-6 \mathrm{wk}$ \\
\hline 13 & Evicore TAAD Panel & 23 & $\begin{array}{l}\text { ACTA2, CBS, COL3A1, COL5A1, COL5A2, EFEMP2, FBN1, } \\
\text { FBN2, FLNA, MED12, MYH11, MYLK, NOTCH1, PLOD1, } \\
\text { PRKG1, SKI, SLC2A10, SMAD3, SMAD4, TGFB2, TGFB3, } \\
\text { TGFBR1, TGFBR2 }\end{array}$ & \\
\hline
\end{tabular}


TABLE 2. Continued

\begin{tabular}{|c|c|c|c|c|}
\hline & Name & $\begin{array}{l}\text { Number } \\
\text { of genes }\end{array}$ & Genetic panel genes & Turnaround time \\
\hline 14 & $\begin{array}{l}\text { Connective Tissue Gene Tests Marfan } \\
\text { Syndrome, Loeys-Dietz } \\
\text { Syndrome, Familial TAAD, and } \\
\text { Related Disorders Panel }\end{array}$ & 28 & $\begin{array}{l}\text { ACTA2, BGN, CBS, COL3A1, COL5A1, COL5A2, FBN1, FBN2, } \\
\text { FLNA, FOXE3, LOX, LTBP3, MAT2A, MED12, MFAP5, MYH11, } \\
\text { MYLK, NOTCH1, PRKG1, SKI, SLC2A10, SMAD2, SMAD3, } \\
\text { SMAD4, TGFB2, TGFB3, TGFBR1, TGFBR2 }\end{array}$ & $2-4 \mathrm{wk}$ \\
\hline 15 & $\begin{array}{l}\text { Mayo Clinic Laboratories Marfan } \\
\text { Syndrome and Related Disorders } \\
\text { Multi-Gene Panel }\end{array}$ & 21 & $\begin{array}{l}\text { ACTA2, CBS, COL3A1, COL5A1, COL5A2, FBN1, FBN2, FLNA, } \\
\text { MFAP5, MYH11, MYLK, NOTCH1, PRKG1, SKI, SLC2A10, } \\
\text { SMAD3, SMAD4, TGFB2, TGFB3, TGFBR1, TGFBR2 }\end{array}$ & $2-4 \mathrm{wk}$ \\
\hline 16 & $\begin{array}{l}\text { Asper Cardiogenetics Familial TAAD } \\
\text { and Related Syndromes NGS Panel }\end{array}$ & 19 & $\begin{array}{l}\text { ACTA2, BGN, COL3A1, COL5A1, FBN1, LOX, MAT2A, MFAP5, } \\
\quad \text { MYH11, MYLK, NOTCH1, PRKG1, SLC2A10, SMAD3, TGFB2, } \\
\quad \text { TGFB3, TGFBR1, TGFBR2, TGFBR3 }\end{array}$ & $6-9 \mathrm{wk}$ \\
\hline 17 & $\begin{array}{l}\text { CeGaT EDS, MFS, LDS, Aortic } \\
\text { Aneurysm and Differential } \\
\text { Diagnoses }\end{array}$ & 49 & $\begin{array}{l}\text { ABCC6, ACTA2, ACVR1, ADAMTS2, ALDH18A1, ATP6VOA2, } \\
\text { ATP6V1A, ATP6V1E1, B3GALT6, B4GALT7, BGN, C1R, C1S, } \\
\text { CBS, CHST14, COL1A1, COL1A2, COL3A1, COL4A1, COL5A1, } \\
\text { COL5A2, DSE, EFEMP2, ELN, FBLN5, FBN1, FBN2, FKBP14, } \\
\text { FOXE3, GORAB, LOX, LTBP4, MFAP5, MYH11, MYLK, } \\
\text { PLOD1, PRDM5, PYCR1, SKI, SLC2A10, SLC39A13, SMAD3, } \\
\text { SMAD4, TGFB2, TGFB3, TGFBR1, TGFBR2, TNXB, ZNF469 }\end{array}$ & $4-6 \mathrm{wk}$ \\
\hline 18 & Color Hereditary Heart Health $\|$ & 30 & $\begin{array}{l}\text { ACTA2, ACTC1, APOB, COL3A1, SC2, DSG2, DSP, FBN1, GLA, } \\
\text { KCNH2, KCNQ1, LDLR, LMNA, MYBPC3, MYH7, MYH11, } \\
\text { MYL2, MYL3, PCSK9, PKP2, PRKAG2, RYR2, SCN5A, SMAD3, } \\
\text { TGFBR1, TGFBR2, TMEM43, TNNI3, TNNT2, TPM1 }\end{array}$ & \\
\hline 19 & $\begin{array}{l}\text { DDC Clinic Molecular Diagnostics } \\
\text { Laboratory Familial TAAD Panel }\end{array}$ & 10 & $\begin{array}{l}\text { ACTA2, FBN1, MYH11, MYLK, SKI, SMAD3, SMAD6, TGFB2, } \\
\quad \text { TGFBR1, TGFBR2 }\end{array}$ & 4 wk or less \\
\hline
\end{tabular}

OHSU, Oregon Health \& Science University; TAAD, thoracic aortic aneurysm or dissection. *Add-on Preliminary-evidence Gene for Aortopathy; HCN4, MAT2A, SMAD6. $\dagger$ Deletion/duplication detection not available for this gene. †Some, or all, of the gene is duplicated in the genome. $§$ The gene has suboptimal coverage (means $<90 \%$ of the gene's target nucleotides are covered at $>20 \times$ with mapping quality score ( $M Q>20$ ) reads), and/or the gene has exons listed under test limitations section that are not included in the panel, as they are not sufficiently covered with high-quality sequence reads. $\ddagger$ or $\S$ The sensitivity to detect variants may be limited in these genes. "Several regions that cannot be reliably assessed with standard target enrichment protocols are not analyzed: $K C N H 2$ exon $4, K C N Q 1$ exon 1, and $T G F B R I$ exon 1 . In $A P O B$, only positions known to impact familial hypercholesterolemia risk are analyzed: only chr2:g.21229159_21229161 (APOB codon 3527) is analyzed. For the LDLR promoter region, the detection of deletions, duplications, and complex structural rearrangements may be limited.References/Links to Panels

1. https://www.invitae.com/en/physician/tests/02301/\#info-panel-assay_information.

2. https://ltd.aruplab.com/Tests/Pub/2006540.

3. https://www.ohsu.edu/lab-services/familial-aneurysm-and-aortopathy-panel.

4. https://blueprintgenetics.com/tests/panels/cardiology/aorta-panel/.

5. https://www.labcorp.com/tests/451432/geneseq-cardio-familial-aortopathy-profile.

6. https://www.genedx.com/wp-content/uploads/2018/04/Info_Sheetcardio_Marfan-TAAD_update-2_20_20.pdf.

7. https://www.ambrygen.com/providers/genetic-testing/12/cardiology/taadnext.

8. https://www.preventiongenetics.com/testInfo $?$ val $=$ Familial + Thoracic + Aortic + Aneurysm + and + Dissection $+\% 28 \mathrm{TAAD} \% 29+$ Panel .

9. http://uwcpdx.org/core-familial-aneurysm-panel/.

10. https://www.fulgentgenetics.com/marfan-taad.

11. https://www.egl-eurofins.com/tests/MM099.

12. http://www.transgenomic.com/labs/cardiology/familion/marfan-taad.html.

13. https://www.evicore.com/-/media/files/evicore/clinical-guidelines/solution/lab-management/healthplan/test-specific-guidelines/taad_panel_testing 2020_v2.pdf.

14. https://www.ctgt.net/panel/marfan-syndrome-loeys-dietz-syndrome-familial-thoracic-aortic-aneurysms-dissections-and.

15. https://www.mayocliniclabs.com/test-catalog/Performance/63029.

16. https://www.asperbio.com/asper-cardiogenetics/familial-thoracic-aortic-aneurysm-and-dissection-and-related-syndromes-ngs-panel.

17. https://www.cegat.de/en/diagnostics/diagnostic-panels/connective-tissue-diseases/\#CTD02.

18. https://support.color.com/en/articles/2393820-what-is-the-color-hereditary-heart-health-test

19. https://www.ddccliniclab.org/test/detail/familial-thoracic-aortic-aneurysms-ngs-panel. 
TABLE 3. Genes among panels 1-19

\begin{tabular}{|c|c|c|c|c|c|c|c|c|c|c|c|c|c|c|c|c|c|c|c|c|}
\hline Gene & 1 & 2 & 3 & 4 & 5 & 6 & 7 & 8 & 9 & 10 & 11 & 12 & 13 & 14 & 15 & 16 & 17 & 18 & 19 & Total \\
\hline АCTA2 & & $\nu$ & $\nu$ & & & 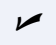 & 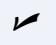 & $\nu$ & $\nu$ & 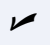 & $\nu$ & $\nu$ & $\nu$ & 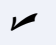 & v & V & $\nu$ & $レ$ & $レ$ & $19(100 \%)$ \\
\hline$B G N$ & & & & $\nu$ & & $レ$ & $\nu$ & & $v$ & $\nu$ & $\nu$ & & & $\nu$ & & $\nu$ & $\nu$ & & & $9(47 \%)$ \\
\hline COL $3 A 1$ & 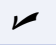 & レ & $\nu$ & $\nu$ & $\nu$ & $\nu$ & $\nu$ & $\nu$ & $\nu$ & $\nu$ & $\nu$ & & $\nu$ & $\nu$ & $\nu$ & $レ$ & $\nu$ & $v$ & & $17(89 \%)$ \\
\hline FBN1 & $\nu$ & $\nu$ & $\nu$ & $\nu$ & $\nu$ & $\nu$ & $\nu$ & $\nu$ & $\nu$ & $\nu$ & $\nu$ & $\nu$ & $\nu$ & 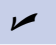 & $v$ & 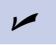 & $\nu$ & $\nu$ & 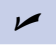 & $19(100 \%)$ \\
\hline FOXE3 & レ & & & 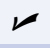 & & & 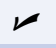 & $\nu$ & レ & 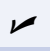 & & & & レ & & & $\nu$ & & & $8(42 \%)$ \\
\hline$L O X$ & & $\nu$ & & $\nu$ & & 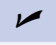 & $\nu$ & $\nu$ & $\nu$ & $\nu$ & & & & $\nu$ & & $\nu$ & $\nu$ & & & $10(53 \%)$ \\
\hline$M A T 2 A$ & & & & $\nu$ & & 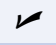 & $\nu$ & $\nu$ & $\nu$ & $\nu$ & & & & $\nu$ & & V & & & & $8(42 \%)$ \\
\hline MFAP5 & & & & $\swarrow$ & & $v$ & $\nu$ & $\nu$ & $\nu$ & $\nu$ & & & & $\nu$ & $\nu$ & $\nu$ & $\nu$ & & & $10(53 \%)$ \\
\hline МYH11 & & $\nu$ & 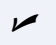 & $\nu$ & レ & $\nu$ & $\nu$ & $\nu$ & $\nu$ & $\nu$ & $\nu$ & & レ & $\nu$ & $\boldsymbol{V}$ & レ & $\nu$ & 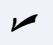 & 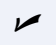 & $18(95 \%)$ \\
\hline$M Y L K$ & 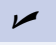 & $\nu$ & $v$ & V & $\nu$ & $\nu$ & $\nu$ & $\nu$ & $\nu$ & $\nu$ & $\nu$ & & $\nu$ & 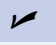 & $\nu$ & $\nu$ & 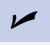 & & 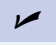 & $17(89 \%)$ \\
\hline PRKG1 & $\boldsymbol{}$ & V & & 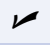 & & 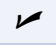 & V & $\nu$ & $\nu$ & $\nu$ & & & $レ$ & V & $\nu$ & 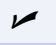 & & & & $12(63 \%)$ \\
\hline$S M A D 3$ & $\nu$ & $v$ & $\nu$ & $\nu$ & $\nu$ & $\nu$ & $\nu$ & $\nu$ & $\nu$ & $\nu$ & $\nu$ & & $\nu$ & $\nu$ & $\nu$ & $\nu$ & $v$ & $\nu$ & $\nu$ & $18(95 \%)$ \\
\hline TGFB2 & レ & $\nu$ & $\nu$ & $\nu$ & $\nu$ & $レ$ & $\nu$ & $\nu$ & レ & $\nu$ & $\nu$ & & 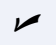 & $\nu$ & $\nu$ & レ & $\nu$ & & 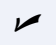 & $17(89 \%)$ \\
\hline TGFB3 & $\nu$ & $\nu$ & 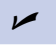 & $\nu$ & & 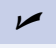 & $\nu$ & $\swarrow$ & $\nu$ & 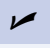 & & & $\nu$ & $\nu$ & $\nu$ & $\boldsymbol{\nu}$ & V & & & $14(74 \%)$ \\
\hline TGFBRI & & $\nu$ & $\nu$ & $\nu$ & 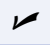 & 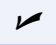 & $\nu$ & $\nu$ & $\nu$ & $\nu$ & $\nu$ & $\nu$ & 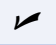 & $\nu$ & レ & レ & $\nu$ & $\swarrow$ & 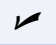 & $19(100 \%)$ \\
\hline TGFBR2 & $レ$ & 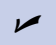 & $\nu$ & $\nu$ & 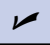 & $\nu$ & 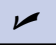 & $v$ & $\nu$ & $\nu$ & $\nu$ & $\nu$ & $\nu$ & $\nu$ & $\nu$ & $\nu$ & $\nu$ & $\nu$ & 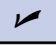 & $19(100 \%)$ \\
\hline
\end{tabular}

Panels 1-19 in top row corresponds to panels 1-19 in the first column of Table 2.

ascending aortic diameter is $>4.0 \mathrm{~cm} .^{3,31}$ However, the different mutations within LDS have different phenotypes, and it has been suggested that a threshold of $>4.0 \mathrm{~cm}$ for prophylactic surgical repair is overly aggressive for patients with a pathogenic variant in $S M A D 3^{32}$ due the dissection occurring at larger aortic diameters; for example, Hostetler and colleagues ${ }^{32}$ found that more than $67 \%$ of patients with SMAD3 variants had aortic roots $>5.0 \mathrm{~cm}$ at time of dissection. In addition, patients with SMAD3 variants had a later onset of aortic events compared with those with TGFBRI and $T G B R 2$ variants and that aortic events in children were rare. ${ }^{32}$ In patients with TGFBRI mutations, female patients have less risk of aortic event than male patients; therefore, we could be less aggressive in female patients with TGFBR1 mutations compared to males. However, among patients with $T G F B R 2$ mutations, female and male patients have similar aortic risk; and, therefore, both male and female patients with TGFBR2 mutations should be managed similarly. ${ }^{33}$

If a pathogenic variant is identified in COL3AI and the diagnosis of vEDS is made, then noninvasive vascular imaging is recommended. In contrast to MFS and LDS, the role of prophylactic surgical repair of unruptured aneurysms remains unclear; however, surgical repair of acute aortic dissections and aortic root aneurysms can be accomplished, although it is complicated due to the friability of tissues. $^{22}$

In nonsyndromic TAAD, specific guidelines regarding surveillance and prophylactic surgical repair for each HTAD gene are lacking. Identification of a pathogenic variant in ACTA2 warrants prophylactic surgical repair when the aortic root or ascending aorta diameter is $\geq 4.5 \mathrm{~cm} .{ }^{34}$ Specific criteria do not exist for other known HTAD genes despite the differing phenotypes. Patients with a PRKG1 mutation are predisposed to an aggressive form of thoracic aortic disease and suffer aortic events at relatively young ages and small aortic diameters. ${ }^{14,35,36}$ Around $30 \%$ of patients with a PRKG1 mutation suffered a type A aortic dissection at an average age of 34 years and an aortic diameter of $5.0 \mathrm{~cm}^{3}$. Around $17 \%$ of patients with a MYH1 1 mutation suffered a type A aortic dissection at an average age of 44 years at an aortic diameter of $4.4 \mathrm{~cm}^{3}$. Therefore, prophylactic aortic repair is reasonable at smaller diameters than non-genetically mediated aortopathy.

\section{EXTENT OF AORTIC REPAIR}

In the setting of genetic aortopathy, once aortic diameter reaches threshold or exceeds a growth rate of $0.5 \mathrm{~cm} /$ year, prophylactic aortic repair should be performed. However, to what extent should aortic repair be done? The aortic root is mainly derived from the second heart field and ascending aorta is derived from neural crest cells and the second heart field in contrast to the descending thoracic and abdominal aorta ${ }^{37}$; therefore, these genetic aortopathies affect the aortic root and ascending aorta differently than the distal aorta.

For prophylactic repair, in regard to the aortic root, aortic root replacement should be performed when the aortic root is $>5.0 \mathrm{~cm}$ in patients with $\mathrm{MFS}^{3}$ and $>4.0 \mathrm{~cm}$ in patients with LDS. ${ }^{22}$ A valve-sparing root replacement should be considered if depending on presence/extent of aortic regurgitation. Ascending aortic replacement should be 


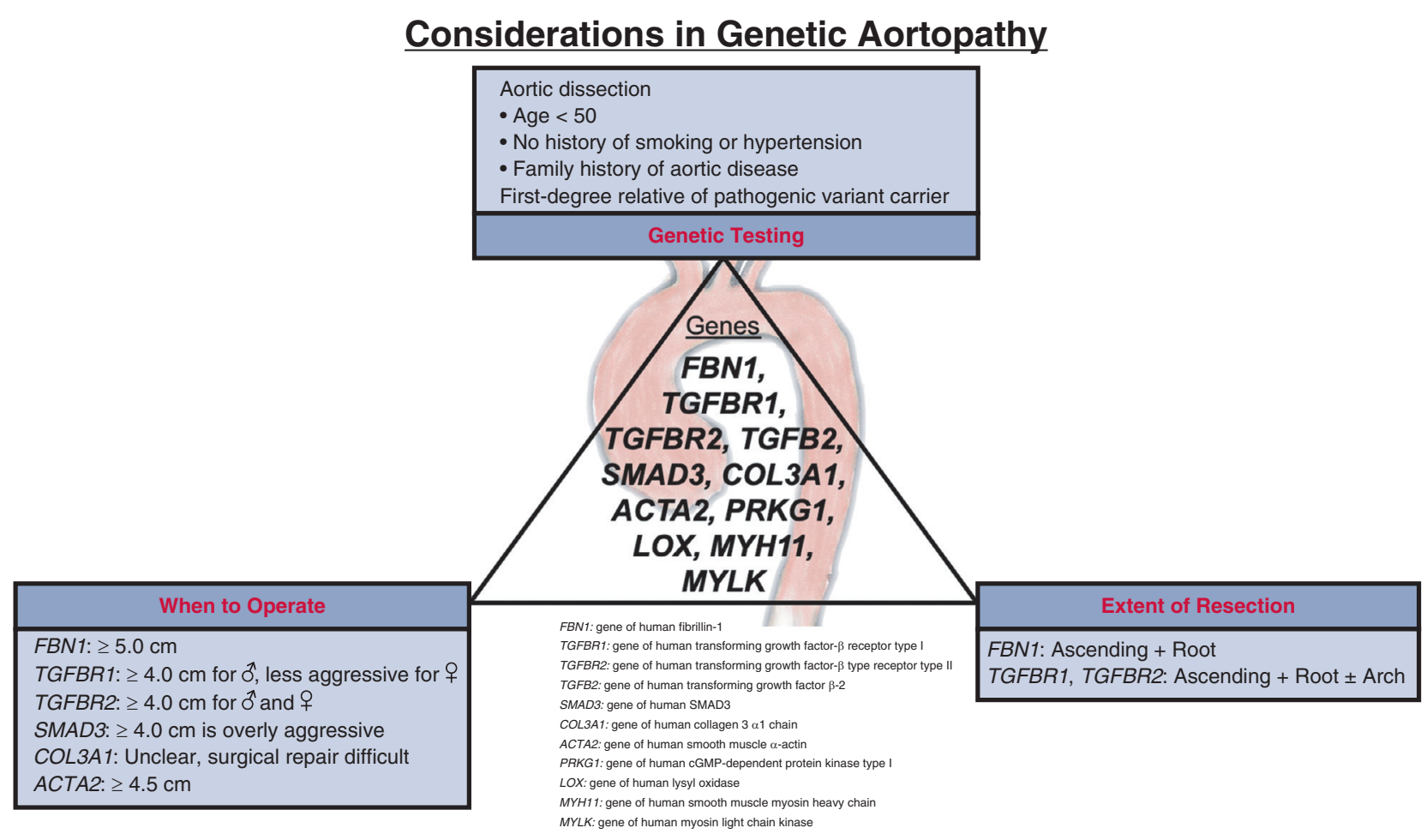

The recognition of genetic aortopathy is critically important in the management of thoracic aortic disease and management should be tailored based on specific causative genetic variant

FIGURE 1. Genetic aortopathy is an important etiology of thoracic aortic aneurysms and dissections and clinical genetic testing should be considered in high-risk patients. Identification of a pathogenic in a known heritable thoracic aortic disease-associated gene should guide clinical decision-making and both when to operate and extent of resection should be tailored to the specific causative gene.

performed based on the same growth rate and diameter thresholds; however, if replacing the root, the ascending should also be replaced in patients with genetic aortopathies to prevent ascending aortic dissection and rupture. In regard to the aortic arch, patients with MFS rarely undergo arch interventions if they have not had a type A aortic dissection; however, patients with LDS require more aortic arch interventions. ${ }^{38}$ Therefore, in patients with MFS, prophylactic replacement of the aortic arch at time of elective root/ ascending repair is not indicated. ${ }^{39}$ It is reasonable to consider prophylactic aortic arch replacement at time of elective root/ascending repair in patients with LDS to prevent subsequent arch interventions. ${ }^{38}$

Despite adequate surveillance, acute dissection can occur at diameters below recommended thresholds. When a patient presents with an acute type A aortic dissection (ATAAD) with a known genetic diagnosis the question regarding extent of repair persists. As with any ATAAD, the ascending aorta should be replaced and both the primary intimal tear and any aneurysmal section should be resected. Patients with pathogenic variants have an increased incidence of reoperation for aortic root pathology ${ }^{40}$ if the aortic root is not replaced; therefore, at time of ATAAD, the aortic root should be replaced in patients with a known or suspected to have genetic mutation. ${ }^{41,42}$ However, in regard to the aortic arch, optimal extent remains controversial. Some recommend an aggressive approach and arch replacement in patients with MFS, ${ }^{43}$ whereas others think a conservative approach is appropriate. ${ }^{44}$ When evaluating extent, the goals of having the patient survive the operation and preventing future reoperations need to be balanced. Yang and colleagues $^{44}$ found that MFS was not a significant risk factor for reoperation (hazard ratio, 1.15); therefore, a diagnosis of MFS alone should not be used as an indication for aggressive arch replacement. Similarly, Schoenhoff and colleagues ${ }^{38}$ found that arch reinterventions following dissection were similar between patients with LDS and MFS; however, patients with LDS had a greater rate of arch intervention after root surgery in the absence of dissection; therefore, if LDS are stable it is reasonable to aggressively replace the aortic arch because of the greater risk of arch reintervention during ATAAD repair. In addition, carrying a pathogenic variant was not a significant risk factor for reoperation of the aortic arch, descending thoracic, or abdominal aorta; however, this was in a population in which FBN1 mutations make up $71 \%$ of the pathogenic 
mutations. ${ }^{40}$ If pathogenic variant status is unknown at time of dissection (often times the case), patients with a high chance of carrying a pathogenic variant (marfanoid habitus, family history of aortic disease, $<50$ years, and no history of smoking or hypertension) should be managed as such. ${ }^{40}$

\section{CONCLUSIONS}

Genetic aortopathy is an important etiology of thoracic aortic aneurysms and dissections and clinical genetic testing could better facilitate clinical practice and prevent the morbidity and mortality associated with acute aortic events. HTAD is a blanket term the encompasses a variety of phenotypes and clinical courses. Therefore, the specific causative gene should be used when determining clinical decisions, specifically surveillance timeframe and modality, when elective aortic repair is indicated, and extent of aortic repair in both elective and emergent settings (Figure 1).

\section{Conflict of Interest Statement}

The authors reported no conflicts of interest.

The Journal policy requires editors and reviewers to disclose conflicts of interest and to decline handling or reviewing manuscripts for which they may have a conflict of interest. The editors and reviewers of this article have no conflicts of interest.

\section{References}

1. Bickerstaff LK, Pairolero PC, Hollier LH, Melton LJ, Van Peenen HJ, Cherry KJ, et al. Thoracic aortic aneurysms: a population-based study. Surgery. 1982;92: 1103-8.

2. Hagan PG, Nienaber CA, Isselbacher EM, Bruckman D, Karavite DJ, Russman PL, et al. The international registry of acute aortic dissection (IRAD): new insights into an old disease. JAMA. 2000;283:897-903.

3. Milewicz DM, Regalado E. Heritable thoracic aortic disease overview. In: Adam MP, Ardinger HH, Pagon RA, Wallace SE, Bean LJH, Stephens K, eds. GeneReviews ${ }^{\circledR}$ [Internet]. Seattle (WA): University of Washington, Seattle; 2017. 1993-2020.

4. Renard M, Francis C, Ghosh R, Scott AF, Witmer PD, Ades LC, et al. Clinical validity of genes for heritable thoracic aortic aneurysm and dissection. J Am Coll Cardiol. 2018;72:605-15.

5. Faggion Vinholo T, Brownstein AJ, Ziganshin BA, Zafar MA, Kuivaniemi H, Body SC, et al. Genes associated with thoracic aortic aneurysm and dissection: 2019 update and clinical implications. Aorta (Stamford). 2019;7:99-107.

6. Pomianowski P, Elefteriades JA. The genetics and genomics of thoracic aortic disease. Ann Cardiothorac Surg. 2013;2:271-9.

7. Pinard A, Jones GT, Milewicz DM. Genetics of thoracic and abdominal aortic diseases. Circ Res. 2019;124:588-606.

8. Fletcher AJ, Syed MBJ, Aitman TJ, Newby DE, Walker NL. Inherited thoracic aortic disease: new insights and translational targets. Circulation. 2020;141: 1570-7.

9. Rohde S, Zafar MA, Ziganshin BA, Elefteriades JA. Thoracic aortic aneurysm gene dictionary. Asian Cardiovasc Thorac Ann. 2020; https://doi.org/10.1177/ 0218492320943800 .

10. Ostberg NP, Zafar MA, Ziganshin BA, Elefteriades JA. The genetics of thoracic aortic aneurysms and dissection: a clinical perspective. Biomolecules. 2020;10:182.

11. Groth KA, Stochholm K, Hove H, Andersen NH, Gravholt CH. Causes of Mortality in the Marfan syndrome (from a nationwide register study). Am J Cardiol. 2018;122:1231-5.

12. Loeys BL, Chen J, Neptune ER, Judge DP, Podowski M, Holm T, et al. A syndrome of altered cardiovascular, craniofacial, neurocognitive and skeletal development caused by mutations in TGFBR1 or TGFBR2. Nat Genet. 2005;37: 275-81.
13. Pepin M, Schwarze U, Superti-Furga A, Byers PH. Clinical and genetic features of Ehlers-Danlos syndrome type IV, the vascular type. N Engl J Med. 2000;342: 673-80.

14. Gago-Diaz M, Blanco-Verea A, Teixido G, Huguet F, Gut M, Laurie S, et al. PRKG1 and genetic diagnosis of early-onset thoracic aortic disease. Eur J Clin Invest. 2016;46:787-94.

15. Jiao J, Xiong W, Wang L, Yang J, Qiu P, Hirai H, et al. Differentiation defect in neural crest-derived smooth muscle cells in patients with aortopathy associated with bicuspid aortic valves. EBioMedicine. 2016;10:282-90.

16. Norton E, Yang B. Managing thoracic aortic aneurysm in patients with bicuspid aortic valve based on aortic root-involvement. Front Physiol. 2017;8:397.

17. Laforest B, Andelfinger G, Nemer M. Loss of Gata5 in mice leads to bicuspid aortic valve. J Clin Invest. 2011;121:2876-87.

18. Gharibeh L, Komati H, Bosse Y, Boodhwani M, Heydarpour M, Fortier M, et al. GATA6 regulates aortic valve remodeling, and its haploinsufficiency leads to right-left type bicuspid aortic valve. Circulation. 2018;138:1025-38.

19. Yang B, Zhou W, Jiao J, Nielsen JB, Mathis MR, Heydarpour M, et al. Proteinaltering and regulatory genetic variants near GATA4 implicated in bicuspid aortic valve. Nat Commun. 2017;8:15481.

20. Gould RA, Aziz H, Woods CE, Seman-Senderos MA, Sparks E, Preuss C, et al. ROBO4 variants predispose individuals to bicuspid aortic valve and thoracic aortic aneurysm. Nat Genet. 2019;51:42-50.

21. Milewicz DM, Ramirez F. Therapies for thoracic aortic aneurysms and acute aortic dissections. Arterioscler Thromb Vasc Biol. 2019;39:126-36.

22. Hiratzka LF, Bakris GL, Beckman JA, Bersin RM, Carr VF, Casey DE Jr, et al. 2010 ACCF/AHA/AATS/ACR/ASA/SCA/SCAI/SIR/STS/SVM guidelines for the diagnosis and management of patients with thoracic aortic disease. Circulation. 2010;121:e266-369.

23. Wolford BN, Hornsby WE, Guo D, Zhou W, Lin M, Farhat L, et al. Clinical implications of identifying pathogenic variants in individuals with thoracic aortic dissection. Circ Genom Precis Med. 2019;12:e002476.

24. Verhagen JMA, Kempers M, Cozijnsen L, Bouma BJ, Duijnhouwer AL, Post JG, et al. Expert consensus recommendations on the cardiogenetic care for patients with thoracic aortic disease and their first-degree relatives. Int J Cardiol. 2018; 258:243-8.

25. Mariscalco G, Debiec R, Elefteriades JA, Samani NJ, Murphy GJ. Systematic review of studies that have evaluated screening tests in relatives of patients affected by nonsyndromic thoracic aortic disease. J Am Heart Assoc. 2018;7:e009302.

26. Demo E, Rigelsky C, Rideout AL, Graf M, Pariani M, Regalado E, et al. Genetics and precision medicine: heritable thoracic aortic disease. Med Clin North Am. 2019;103:1005-19.

27. Overwater E, Marsili L, Baars MJH, Baas AF, van de Beek I, Dulfer E, et al. Results of next-generation sequencing gene panel diagnostics including copynumber variation analysis in 810 patients suspected of heritable thoracic aortic disorders. Hum Mutat. 2018;39:1173-92.

28. Hicks KL, Byers PH, Quiroga E, Pepin MG, Shalhub S. Testing patterns for genetically triggered aortic and arterial aneurysms and dissections at an academic center. J Vasc Surg. 2018;68:701-11.

29. Dawson A, LeMaire SA. Building on a genetic framework: can we personalize the timing of surgical repair for patients with heritable thoracic aortic disease? J Thorac Cardiovasc Surg. 2020;160:901-5.

30. Ouzounian M, LeMaire SA. How can genetic diagnosis inform the decision of when to operate? J Vis Surg. 2018;4:68.

31. MacCarrick G, Black JH III, Bowdin S, El-Hamamsy I, FrischmeyerGuerrerio PA, Guerrerio AL, et al. Loeys-Dietz syndrome: a primer for diagnosis and management. Genet Med. 2014;16:576-87.

32. Hostetler EM, Regalado ES, Guo DC, Hanna N, Arnaud P, Muino-Mosquera L, et al. SMAD3 pathogenic variants: risk for thoracic aortic disease and associated complications from the Montalcino Aortic Consortium. J Med Genet. 2019;56:252-60.

33. Jondeau G, Ropers J, Regalado E, Braverman A, Evangelista A, Teixedo G, et al. International registry of patients carrying TGFBR1 or TGFBR2 Mutations: results of the MAC (Montalcino Aortic Consortium). Circ Cardiovasc Genet. 2016;9:548-58.

34. Regalado ES, Guo DC, Prakash S, Bensend TA, Flynn K, Estrera A, et al. Aortic disease presentation and outcome associated with ACTA2 Mutations. Circ Cardiovasc Genet. 2015;8:457-64.

35. Guo DC, Regalado E, Casteel DE, Santos-Cortez RL, Gong L, Kim JJ, et al. Recurrent gain-of-function mutation in PRKG1 causes thoracic aortic aneurysms and acute aortic dissections. Am J Hum Genet. 2013;93:398-404.

36. Norton EL, Gordon D, Yang B. Managing the aorta in patients with a PRKG1 mutation. J Thorac Cardiovasc Surg. 2019;157:e107-9.

37. Sawada H, Rateri DL, Moorleghen JJ, Majesky MW, Daugherty A. Smooth muscle cells derived from second heart field and cardiac neural crest reside in 
spatially distinct domains in the media of the ascending aorta-brief report. Arterioscler Thromb Vasc Biol. 2017;37:1722-6.

38. Schoenhoff FS, Alejo DE, Black JH, Crawford TC, Dietz HC, Grimm JC, et al. Management of the aortic arch in patients with Loeys-Dietz syndrome. J Thorac Cardiovasc Surg. 2020;160:1166-75.

39. Schoenhoff FS, Kadner A, Czerny M, Jungi S, Meszaros K, Schmidli J, et al. Should aortic arch replacement be performed during initial surgery for aortic root aneurysm in patients with Marfan syndrome? Eur J Cardiothorac Surg. 2013;44:346-51; discussion 351.

40. Norton EL, Hornsby WE, Wu X, Wolford BN, Graham SE, Willer CJ, et al. Aortic progression and reintervention in patients with pathogenic variants after a thoracic aortic dissection. J Thorac Cardiovasc Surg. February 20, 2020 [Epub ahead of print].

41. Rylski B, Bavaria JE, Beyersdorf F, Branchetti E, Desai ND, Milewski RK, et al. Type A aortic dissection in Marfan syndrome: extent of initial surgery determines long-term outcome. Circulation. 2014;129:1381-6.
42. Yang B, Norton EL, Hobbs R, Farhat L, Wu X, Hornsby WE, et al. Short- and long-term outcomes of aortic root repair and replacement in patients undergoing acute type A aortic dissection repair: 20-year experience. J Thorac Cardiovasc Surg. 2019;157:2125-36.

43. Bachet J, Larrazet F, Goudot B, Dreyfus G, Folliguet T, Laborde F, et al. When should the aortic arch be replaced in Marfan patients? Ann Thorac Surg. 2007;83: S774-9; discussion S785-90.

44. Yang B, Norton EL, Shih T, Farhat L, Wu X, Hornsby WE, et al. Late outcomes of strategic arch resection in acute type A aortic dissection. J Thorac Cardiovasc Surg. 2019;157:1313-21.e2.

Key Words: thoracic aortic aneurysm, aortic dissection, aortopathy, genetics, Marfan syndrome, Loeys-Dietz syndrome, heritable thoracic aortic disease 